\title{
ON THE EXISTENCE OF STRONGLY SERIES SUMMABLE MARKUSCHEVICH BASES IN BANACH SPACES
}

\author{
BY
}

WILLIAM B. JOHNSON

\begin{abstract}
The main result is: Let $X$ be a complex separable Banach space. If the identity operator on $X^{*}$ is the limit in the strong operator topology of a uniformly bounded net of linear operators of finite rank, then $X$ admits a strongly series summable Markuschevich basis.
\end{abstract}

I. Introduction. Let $X$ be a separable Banach space. A biorthogonal sequence $\left\{x_{i}, f_{i}\right\}_{i=1}^{\infty}$ in $\left(X, X^{*}\right)$ is called a Markuschevich basis ( $M$-basis) for $X$ provided $\left\{x_{i}\right\}_{i=1}^{\infty}$ is fundamental in $X$ and $\left\{f_{i}\right\}_{i=1}^{\infty}$ is total over $X$. Following Ruckle [8], we say that an $M$-basis $\left\{x_{i}, f_{i}\right\}_{i=1}^{\infty}$ for $X$ is strongly series summable (s.s.s.) provided there exists a set $\left\{\lambda_{i, n}: i=1,2, \ldots, n ; n=1,2, \ldots\right\}$ of scalars (called a summation matrix for $\left.\left\{x_{i}, f_{i}\right\}_{i=1}^{\infty}\right)$ such that, for each $x$ in $X, x=\lim _{n \rightarrow \infty} \sum_{i=1}^{n} \lambda_{i, n} f_{i}(x) x_{i}$. Note that a Schauder basis is a s.s.s. $M$-basis for which each $\lambda_{i, n}$ can be chosen to be 1 .

The results of [8], [9], and [4] indicate that the duality theory of a space which has a s.s.s. $M$-basis is essentially the same as that of a space which admits a Schauder basis. The reason for this appears to be that if $\left\{x_{i}, f_{i}\right\}_{i=1}^{\infty}$ is a s.s.s. $M$-basis for $X$ with summation matrix $\left(\lambda_{i, n}\right)$ then, for each $f$ in the coefficient space $\left({ }^{1}\right)$ of the basis, $f$ is the norm limit of $\left\{\sum_{i=1}^{n} \lambda_{i, n} f\left(x_{i}\right) f_{i}\right\}_{n=1}^{\infty}$. Thus the adjoints of the "partial sum" operators defined by $T_{n}(x)=\sum_{i=1}^{n} \lambda_{i, n} f_{i}(x) x_{i}$ also act like partial sum operators. In this respect s.s.s. $M$-bases behave more like Schauder bases than do such weaker structures as generalized summation bases (see [3]).

In this paper we prove the following rather strong existence theorem for s.s.s. $M$-bases:

THEOREM 1. Let $X$ be a separable complex Banach space such that $X^{*}$ has the $\lambda$-metric approximation property for some $\lambda \geqq 1$. If $Y$ is a separable subspace of $X^{*}$, then there exists a strongly series summable Markuschevich basis for $X$ whose coefficient space contains $Y$.

If $\lambda \geqq 1$, we say that the Banach space $X$ has the $\lambda$-metric approximation property $\left(\lambda\right.$-m.a.p.) if there is a net $\left\{S_{d}: d \in D\right\}$ of linear operators of finite rank on $X$

Received by the editors April 20, 1970.

AMS 1969 subject classifications. Primary 4610.

Key words and phrases. Strongly series summable Markuschevich bases, complete biorthogonal sequences, Schauder bases, metric approximation property.

(1) The coefficient space of an $M$-basis $\left\{x_{i}, f_{i}\right\}_{t=1}^{\infty}$ for $X$ is the norm closure in $X^{*}$ of the linear span of $\left\{f_{i}\right\}_{i=1}^{\infty}$. 
uniformly bounded by $\lambda$ which converges pointwise (i.e., in the strong operator topology) to the identity operator on $X$. Equivalently, $X$ has the $\lambda$-m.a.p. provided that, for each finite-dimensional subspace $F$ of $X$ and positive number $\varepsilon$, there is an operator $S$ of finite rank on $X$ such that $\|S\| \leqq \lambda$ and $\|S(x)-x\| \leqq \varepsilon\|x\|$ for each $x \in F$.

The 1-m.a.p. was introduced by Grothendieck [2] under the name metric approximation property. Grothendieck showed that if $X$ is reflexive and has the (topological) approximation property, then in fact both $X$ and $X^{*}$ have the 1-m.a.p. This result together with Theorem 1 implies that every separable, reflexive complex Banach space which has the approximation property also admits a s.s.s. $M$-basis. Of course, it may be that Theorem 1 is always applicable, for it is not even known that there exists a Banach space which does not have the 1-m.a.p.

We use the following notation: $X$ represents a complex Banach space and $X^{*}$ is the dual to $X$. The complex assumption is used in an essential way in Lemma 4, and we do not know whether the real version of Theorem 1 is true. $I$ denotes the identity operator on either $X$ or $X^{*}$. "Operator" means "bounded linear operator". The range space and null space of an operator, $L$, are denoted by, respectively, $\mathscr{R}(L)$ and $\operatorname{ker} L$. If $L$ is an operator on $X$ and $S$ is a subspace of $X$, $L_{\mid S}$ denotes the restriction of $L$ to $S$. The linear span of a subset, $A$, of a linear space is denoted by $\operatorname{sp} A$. The canonical embedding of $X$ into $X^{* *}$ is denoted by “^,".

II. The existence theorem. Our first lemma is both a generalization and a special case of Helly's theorem [11, p. 103].

Lemma 1. Let $F$ be a finite-dimensional Banach space, $S$ a finite-dimensional subspace of $X^{*}, L$ an operator from $X^{*}$ into $F$, and $\varepsilon>0$. There exists a weak*continuous operator $T$ from $X^{*}$ into $F$ such that $T_{1 S}=L_{\mid S}$ and $\|T\| \leqq\|L\|+\varepsilon$.

Proof. We use the notation of [10] in this proof. We identify the weak*continuous operators from $X^{*}$ to $F$ with $X \otimes_{\lambda} F$ and the operators from $X^{*}$ to $F$ with $X^{* *} \otimes_{\lambda} F\left[10\right.$, p. 30]. Since $F$ is finite dimensional, $X^{* *} \otimes_{\lambda} F$ is thereby identified with $\left(X \otimes_{\lambda} F\right)^{* *}$. Now $S \otimes F^{*}$ is identified with a (finite-dimensional) subspace of $\left(X \otimes_{\lambda} F\right)^{*}$, so by Helly's theorem [11, p. 103], there is $T$ in $X \otimes_{\lambda} F$ such that $\|T\| \leqq\|L\|+\varepsilon$ and $f(T(s))=f(L(s))$ for each $s \in S$ and $f \in F^{*}$. Since $F^{*}$ is total over $F, T(s)=L(s)$ for each $s \in S$ and hence $T_{\mid S}=L_{\mid S}$. Q.E.D.

A Banach space $X$ is said to have the $\lambda$ duality metric approximation property $(\lambda \geqq 1)$ provided there is a net $\left\{S_{d}: d \in D\right\}$ of operators of finite rank on $X$ uniformly bounded by $\lambda$ such that $\left\{S_{d}: d \in D\right\}$ converges pointwise to $I$ and $\left\{S_{d}^{*}: d \in D\right\}$ converges pointwise to $I$. Equivalently, $X$ has the $\lambda$ duality m.a.p. provided that, for each $\varepsilon>0$ and each pair of finite-dimensional subspaces $E$ of $X$ and $F$ of $X^{*}$, there is an operator $L$ of finite rank on $X$ such that $\|L\| \leqq \lambda,\|L(x)-x\| \leqq \varepsilon\|x\|$ for each $x \in E$, and $\left\|L^{*}(f)-f\right\| \leqq \varepsilon\|f\|$ for each $f \in F$.

Lemma 2. Suppose that $X^{*}$ has the $\lambda$-m.a.p. Then $X$ has the $\lambda$ duality m.a.p. 
Proof. Using the hypothesis and Lemma 1, we can construct a net $\left\{S_{d}: d \in D\right\}$ of operators of finite rank on $X$ uniformly bounded by $\lambda$ such that $\left\{S_{d}^{*}: d \in D\right\}$ is pointwise convergent on $X^{*}$ to $I$. For each $x \in X$, the net $\left\{S_{d}(x): d \in D\right\}$ weakly converges to $x$, hence (cf., e.g., [1, p. 477]) there is a net $\left\{T_{e}: e \in E\right\}$ of operators on $X$ such that $\left\{T_{e}: e \in E\right\}$ is pointwise convergent on $X$ to $I$; each $T_{e}$ is a convex combination of a subset $\left\{S_{e(i)}\right\}_{i=1}^{n_{e}}$ of $\left\{S_{d}: d \in D\right\}$; and for each $d \in D$ there is $e^{\prime} \in E$ such that if $e \geqq e^{\prime}$ then $e(i) \geqq d$ for $i=1,2, \ldots, n_{e}$. Thus $\left\{T_{e}: e \in E\right\}$ is uniformly bounded by $\lambda$ and $\left\{T_{e}^{*}: e \in E\right\}$ is pointwise convergent on $X^{*}$ to $I$. Q.E.D.

The proof of the next lemma is suggested by the proof of Lemma 3.1 of [5].

Lemma 3. Suppose that $X$ has the $\lambda$ duality m.a.p., $E$ is a finite-dimensional subspace of $X, F$ is a finite-dimensional subspace of $X^{*}$, and $\varepsilon>0$. Then there exists an operator $L$ of finite rank on $X$ such that $\|L\| \leqq \lambda+\varepsilon, L_{\mid E}=I_{\mid E}$, and $L_{\mid F}^{*}=I_{\mid F}$.

Proof. Let $n=\operatorname{dim} E$ and $m=\operatorname{dim} F$. Choose $1>\beta>0$ small enough so that $\beta+\beta m(\lambda+\beta) /(1-\beta) \leqq \varepsilon$ and choose $1>\alpha>0$ small enough so that $(n \alpha /(1-\alpha)) \lambda \leqq \beta / 2$.

Let $M$ be an operator of finite rank on $X$ such that $\|M\| \leqq \lambda$ and, for each $x \in E$ and $f \in F$,

(1) $\|x-M(x)\| \leqq \alpha\|x\|$ and

(2) $\left\|f-M^{*}(f)\right\| \leqq \beta / 2\|f\|$.

By (1), for each $x \in E,(1-\alpha)\|x\| \leqq\|M(x)\|$, hence $M_{\mid E}$ has an inverse, $Q$, satisfying $\|Q\| \leqq 1 /(1-\alpha)$. Also, for each $y \in M[E],\|Q(y)-y\| \leqq(\alpha /(1-\alpha))\|y\|$.

Let $P$ be a projection of $X$ onto $M[E]$ such that $\|P\| \leqq n$. Let $N=Q P M+(I-P) M$. Clearly $N$ has finite rank and $N_{\mid E}=I_{\mid E}$. Now if $x \in X$,

$$
\|N(x)-M(x)\|=\|Q P M(x)-P M(x)\| \leqq \frac{\alpha}{1-\alpha}\|P\|\|M\|\|x\| \leqq \frac{n \alpha \lambda}{1-\alpha}\|x\| .
$$

Thus $\|N-M\| \leqq n \alpha \lambda /(1-\alpha)$, from which it follows that $\left\|N^{*}\right\| \leqq \lambda+\beta / 2$ and $\left\|N^{*}-M^{*}\right\| \leqq \beta / 2$. This last inequality and (2) imply that, for each $f \in F$, $\left\|N^{*}(f)-f\right\| \leqq \beta\|f\|$.

As in the first part of the proof, we have that $N_{\mid F}^{*}$ is an isomorphism with inverse, $Q^{\prime}$, satisfying $\left\|Q^{\prime}(f)-f\right\| \leqq(\beta /(1-\beta))\|f\|$ for each $f \in N^{*}[F]$. Let $P^{\prime}$ be a projection of $X^{*}$ onto $N^{*}[F]$ such that $\left\|P^{\prime}\right\| \leqq m$ and let $L^{*}=Q^{\prime} P^{\prime} N^{*}+\left(I-P^{\prime}\right) N^{*}$. (Note that $L^{*}$ is indeed weak*-continuous because $N^{*}$ is weak*-continuous and has finite rank.) Then $L_{\mid F}^{*}=I_{\mid F}$ and, for each $f \in X^{*}$,

$$
\left\|L^{*}(f)-N^{*}(f)\right\| \leqq \frac{\beta}{1-\beta}\left\|P^{\prime}\right\|\left\|N^{*}\right\|\|f\| \leqq \frac{\beta m(\lambda+\beta)}{1-\beta}\|f\| .
$$

Thus $\left\|L^{*}\right\| \leqq \lambda+\beta+\beta m(\lambda+\beta) /(1-\beta) \leqq \lambda+\varepsilon$.

Since $\|L\|=\left\|L^{*}\right\|$, it remains to be seen only that $L_{\mid E}=I_{\mid E}$. Let $x \in E$ and suppose that $f \in X^{*}$. Then using the fact that $x=N(x)$, we have

$$
\begin{aligned}
f(L(x)) & =L^{*}(f)(x)=L^{*}(f)(N(x)) \\
& =N^{*} Q^{\prime} P^{\prime} N^{*}(f)(x)+N^{*}\left(I-P^{\prime}\right) N^{*}(f)(x) \\
& =P^{\prime} N^{*}(f)(x)+f(N(N(x)))-P^{\prime} N^{*}(f)(N(x))=f(x) .
\end{aligned}
$$

Since $X^{*}$ is total over $E, L(x)=x$. Q.E.D. 
Lemma 4. Let $\left\{x_{i}, f_{i}\right\}_{i=1}^{n}$ be a finite biorthogonal set in $\left(X, X^{*}\right)$, let $T$ be an operator of finite rank on $X$ such that $T\left(x_{i}\right)=x_{i}$ and $T^{*}\left(f_{i}\right)=f_{i}$ for $i=1,2, \ldots, n$, and let $\varepsilon>0$. Then there exists a finite biorthogonal set $\left\{x_{i}, f_{i}\right\}_{i=n+1}^{n+m}$ in $\left(X, X^{*}\right)$ and a set $\left\{\lambda_{i}\right\}_{i=1}^{m}$ of complex numbers such that $\left\{x_{i}, f_{i}\right\}_{i=1}^{n+m}$ is biorthogonal and $\|L-T\| \leqq \varepsilon$, where $L$ is the operator on $X$ defined by

$$
L(x)=\sum_{i=1}^{n} f_{i}(x) x_{i}+\sum_{i=n+1}^{n+m} \lambda_{i-n} f_{i}(x) x_{i} .
$$

Proof. Define a projection $U$ on $X$ by $U(x)=\sum_{i=1}^{n} f_{i}(x) x_{i}$ and let $X_{0}=\mathscr{R}(I-U)$. Note that $T U=U T=U$, so $T\left[X_{0}\right] \subset X_{0}$ and $\operatorname{ker} T \subset X_{0}$. Let $P$ be a projection of finite rank on $X_{0}$ such that $P T(I-U)=T P(I-U)=T(I-U)$. (For example, choose ker $P$ to be a closed complement in $\operatorname{ker} T$ to $\mathscr{R}(T) \cap \operatorname{ker} T$ and choose $\mathscr{R}(P)$ to be a complement in $X_{0}$ to ker $P$ which contains $\mathscr{R}(T) \cap X_{0}$.)

Let $m=\operatorname{dim} \mathscr{R}(P)$ and choose a basis $\left\{z_{i}\right\}_{i=1}^{m}$ for $\mathscr{R}(P)$ such that the matrix representation $\left(\alpha_{i j}\right)_{i, j=1}^{m}$ of $T_{\mid \mathscr{R}(P)}$ with respect to $\left\{z_{i}\right\}_{i=1}^{m}$ is lower triangular-i.e., $\alpha_{i j}=0$ if $j>i$. Now pick a sequence $\left\{\lambda_{i}\right\}_{i=1}^{m}$ of pairwise distinct complex numbers sufficiently close to $\left\{\alpha_{i i}\right\}_{i=1}^{m}$ so that

$$
\left\|Q-T_{\mid \mathscr{R}(P)}\right\| \leqq \varepsilon /\|P\|\|I-U\|,
$$

where $Q$ is the operator on $\mathscr{R}(P)$ whose matrix representation, $\left(\beta_{i j}\right)$, with respect to $\left\{z_{i}\right\}_{i=1}^{m}$ is given by

$$
\begin{aligned}
& \beta_{i j}=\lambda_{i} \quad \text { if } i=j, \\
& =\alpha_{i j} \text { if } i \neq j \text {. }
\end{aligned}
$$

Since $\left(\beta_{i j}\right)$ is lower triangular, $\left\{\lambda_{i}\right\}_{i=1}^{m}$ is the set of eigenvalues for $Q$. The $\lambda_{i}$ 's are distinct, so there is a basis $\left\{x_{i}\right\}_{i=n+1}^{n+m}$ for $\mathscr{R}(P)$ such that $Q\left(x_{i}\right)=\lambda_{i-n} x_{i}$ for $i=n+1, \ldots, n+m$. Picking $\left\{f_{i}\right\}_{i=n+1}^{n+m}$ in $\mathscr{R}\left([P(I-U)]^{*}\right)$ biorthogonal to $\left\{x_{i}\right\}_{i=n+1}^{n+m}$, we have that, for each $x \in \mathscr{R}(P), Q(x)=\sum_{i=n+1}^{n+m} \lambda_{i-n} f_{i}(x) x_{i}$.

Now $\left\{x_{i}, f_{i}\right\}_{i=1}^{n+m}$ is biorthogonal and if $L$ is defined by

$$
L(x)=\sum_{i=1}^{n} f_{i}(x) x_{i}+\sum_{i=n+1}^{n+m} \lambda_{i-n} f_{i}(x) x_{i},
$$

then clearly $L=T U+Q P(I-U)$. Thus

$$
\begin{aligned}
\|L-T\| & =\|T U+Q P(I-U)-T U-T P(I-U)\| \\
& \leqq\left\|Q-T_{\mid \mathscr{R}(P)}\right\|\|P\|\|I-U\| \leqq \varepsilon .
\end{aligned}
$$

Proof of Theorem 1. Let $\lambda$ be such that $X$ has the $\lambda$ duality m.a.p. (Lemma 2). Let $\left\{z_{i}\right\}_{i=1}^{\infty}$ be fundamental in $X$ and let $\left\{g_{i}\right\}_{i=1}^{\infty}$ be a subset of $X^{*}$ such that $Y$ is contained in the closure of the linear span of $\left\{g_{i}\right\}_{i=1}^{\infty}$. Assume, without loss of generality, that $\left\|z_{1}\right\|=\left\|g_{1}\right\|=g_{1}\left(z_{1}\right)=1$. We define the desired s.s.s. $M$-basis $\left\{x_{i}, f_{i}\right\}_{i=1}^{\infty}$ for $X$ and a summability matrix $\left(\lambda_{i, n}\right)$ for $\left\{x_{i}, f_{i}\right\}_{i=1}^{\infty}$ by induction. Set $k(1)=1, \quad x_{1}=z_{1}, \quad f_{1}=g_{1}, \quad \lambda_{1,1}=1$. Now suppose $k(m), \quad\left\{x_{i}, f_{i}\right\}_{i=1}^{k(m)}$, and 
$\left\{\lambda_{i, n}: i \leqq n ; n=1,2, \ldots, k(m)\right\}$ have been defined. Extend $\left\{x_{i}, f_{i}\right\}_{i=1}^{k(m)}$ to a biorthogonal set $\left\{x_{i}, f_{i}\right\}_{i=1}^{j}(j=k(m), k(m)+1$, or $k(m)+2)$ so that $z_{m+1} \in \operatorname{sp}\left\{x_{i}\right\}_{i=1}^{j}$ and $g_{m+1} \in \operatorname{sp}\left\{f_{i}\right\}_{i=1}^{j}$ (cf., e.g., the proof of Theorem III.1 in [3]). Now by Lemma 3 and Lemma 4 there are a positive integer $k(m+1) \geqq j$, a biorthogonal set $\left\{x_{i}, f_{i}\right\}_{i=j+1}^{k(m+1)}$ in $\left(X, X^{*}\right)$, and complex numbers $\left\{\alpha_{i}\right\}_{i=j+1}^{k(m+1)}$ such that $\left\{x_{i}, f_{i}\right)_{i=1}^{k(m+1)}$ is biorthogonal and if $T$ is defined on $X$ by $T(x)=\sum_{i=1}^{j} f_{i}(x) x_{i}+\sum_{i=j+1}^{k(m+1)} \alpha_{i} f_{i}(x) x_{i}$, then $\|T\| \leqq \lambda+1 / m$. We complete the induction by defining

$$
\begin{aligned}
\lambda_{i, n} & =\lambda_{i, k(m)} & & \text { if } i \leqq k(m)<n<k(m+1), \\
& =0 & & \text { if } k(m)<i \leqq n<k(m+1), \\
& =1 & & \text { if } i \leqq k(m) \text { and } n=k(m+1), \\
& =\alpha_{i} & & \text { if } k(m)<i \leqq k(m+1)=n .
\end{aligned}
$$

It is easy to check that $\left\{x_{i}, f_{i}\right\}_{i=1}^{\infty}$ has the desired properties. Q.E.D.

REMARK 1. Suppose that $X$ is separable and $X^{*}$ has the $\lambda$-m.a.p. for some $\lambda$. Theorem 1 shows that there are s.s.s. $M$-bases for $X$ whose coefficient spaces are "arbitrarily large". One might guess that if $Y$ is a separable subspace of $X^{*}$ and $Y$ contains a subspace which is the coefficient space of some s.s.s. $M$-basis for $X$, then $Y$ is itself the coefficient space for some s.s.s. $M$-basis for $X$, because the corresponding statement for generalized summation bases is true (cf. [3, proof of Theorem IV.1]). This is not the case: Let $X=l_{1}$. It is a rather easy consequence of Theorem 4.3 of [6] that the coefficient space of any s.s.s. $M$-basis for $l_{1}$ is an $\mathscr{L}_{\infty}$ space in the sense of [6]. Simply pick $Y$ to be a separable subspace of $l_{\infty}\left(=l_{1}^{*}\right)$ which contains $c_{0}$ but is not an $\mathscr{L}_{\infty}$ space. (For example, $Y$ can be the closed span of $c_{0} \cup K$, where $K$ is a subspace of $l_{\infty}$ isomorphic to $l_{2}$. It follows from Theorem 1 of [7] that $Y$ is isomorphic to $c_{0} \oplus l_{2}$ and is thus not an $\mathscr{L}_{\infty}$ space.)

Recall that an $M$-basis $\left\{x_{i}, f_{i}\right\}_{i=1}^{\infty}$ for $X$ whose coefficient space is $X^{*}$ is called shrinking (see [3]). Now if $\left\{x_{i}, f_{i}\right\}_{i=1}^{\infty}$ is a s.s.s. $M$-basis then the remarks in the introduction show that $\left\{f_{i}, \hat{x}_{i}\right\}_{i=1}^{\infty}$ is a s.s.s. $M$-basis for the coefficient space of the basis. Thus a shrinking $M$-basis $\left\{x_{i}, f_{i}\right\}_{i=1}^{\infty}$ which is s.s.s. is also shrinking as a s.s.s. $M$-basis in the sense that $\left\{f_{i}, \hat{x}_{i}\right\}_{i=1}^{\infty}$ is a s.s.s. $M$-basis for $X^{*}$. In view of Theorem 1 , we thus have

Corollary 1. If $X^{*}$ is separable and has the $\lambda$-m.a.p. for some $\lambda \geqq 1$, then $X$ admits a shrinking s.s.s. M-basis.

Let us say that a s.s.s. $M$-basis $\left\{x_{i}, f_{i}\right\}_{i=1}^{\infty}$ is boundedly complete provided there is a summability matrix $\left(\lambda_{i, n}\right)$ for $\left\{x_{i}, f_{i}\right\}_{i=1}^{\infty}$ such that for every sequence $\left\{t_{i}\right\}_{i=1}^{\infty}$ of scalars, if $\left\{\sum_{i=1}^{n} t_{i} \lambda_{i, n} x_{i}\right\}_{n=1}^{\infty}$ is bounded then it is convergent. A simple modification of Theorem II.3 of [3] shows that a s.s.s. $M$-basis is boundedly complete if and only if it is boundedly complete as an $M$-basis in the sense of [3] (and thus in the above definition of boundedly complete "there is a summability matrix" can be replaced by "for each summability matrix"). Thus using Corollary 1 and the results of [3] we have 
THEOREM 2. $X$ admits a boundedly complete s.s.s. $M$-basis if and only if $X$ has the $\lambda$-m.a.p. for some $\lambda \geqq 1$ and $X$ is isomorphic to a separable conjugate Banach space.

We conclude with a conjecture which, by Theorem 2 , has an affirmative answer if $X$ is a conjugate space:

Conjecture 1. If $X$ is separable and has the $\lambda$-m.a.p. for some $\lambda \geqq 1$, then $X$ admits a s.s.s. $M$-basis.

\section{REFERENCES}

1. N. Dunford and J. T. Schwartz, Linear operators. I: General theory, Pure and Appl. Math., vol. 7, Interscience, New York, 1958. MR 22 \#8302.

2. A. Grothendieck, Produits tensoriels topologiques et espaces nucléaires, Mem. Amer. Math. Soc. No. 16 (1955). MR 17, 763.

3. W. B. Johnson, Markuschevich bases and duality theory, Trans. Amer. Math. Soc. 149 (1970), 171-177.

4. —_, Markuschevich bases and duality theory. II (in preparation).

5. J. Lindenstrauss, Extension of compact operators, Mem. Amer. Math. Soc. No. 48 (1964). MR 31 \#3828.

6. J. Lindenstrauss and H. P. Rosenthal, The $\mathscr{L}_{p}$ spaces, Israel J. Math. 8 (1970).

7. H. P. Rosenthal, On totally incomparable Banach spaces, J. Functional Analysis 4 (1969), 167-175. MR 40 \#1758.

8. W. H. Ruckle, Representation and series summability of complete biorthogonal sequences, Pacific J. Math. 34 (1970), 511-528.

9. - The tensor product of complete biorthogonal sequences, Clemson University Technical Report \#25, Clemson, S.C., 1969.

10. R. Schatten, A theory of cross-spaces, Ann. of Math. Studies, no. 26, Princeton Univ. Press, Princeton, N. J., 1950. MR 12, 186.

11. A. Wilanski, Functional analysis, Blaisdell, Waltham, Mass., 1964. MR 30 \#25.

$$
\begin{aligned}
& \text { University OF Houston, } \\
& \text { Houston, TeXas } 77004
\end{aligned}
$$

